\title{
De la flaque méditerranéenne à l'Océan du Tout-Monde: Imaginaires et identités poétiques transatlantiques
}

\author{
May Chehab \\ Université de Chypre
}

Résumé: Grâce à la médiation du celticisme littéraire et au prisme de la sémiotique des couleurs, l'étude examine le passage, dans la littérature francophone du XXe siècle, d'une culture dominée par la Méditerranée à un paradigme littéraire atlantique. L'insularité idéalisée grecque de couleur bleue est remplacée par l'archipel vert des Caraïbes. Dans ce procès une identité atlantique transnationale nouvelle émerge de l'océan de la littérature.

Mots-clés: Celtisme, Saint-John Perse, Glissant, méditerranée, antilles, antiquité, sémiotique de la couleur

\begin{abstract}
Through the mediation of literary Celticism and the perspective of colour semiotics, the paper examines the shift, in the French speaking literature of the twentieth century, from a Mediterraneandominated culture to an Atlantic literary paradigm. The Greek idealized blue insularity is replaced by the green Caribbean Archipelago. In the process, a new transnational Atlantic identity emerges from the sea of literature.
\end{abstract}

Keywords: Celtism, Atlantism, Saint-John Perse, Glissant, mediterranean, caribbean, antiquity, colour semiotics 


\section{Préludes: la Méditerranée et la désaffection de l’antique}

Comme naguère la Méditerranée, l'Atlantique littéraire métaphorise un discours identitaire. En réaction contre une mer gréco-romaine ou dans son fécond prolongement, ce discours prend acte du passage d'une européanité repliée sur elle-même et sur son passé vers une transatlanticité ouverte sur le monde et sur l'à-venir. Au tournant du XXe siècle, plusieurs auteurs de la littérature française en viennent à contester ouvertement le monopole du legs antique et revendiquent, pour certains, une nouvelle filiation. Mais l'évolution des représentations faisant de la Méditerranée la mer originelle de la poésie, dans la plus parfaite tradition hégélienne, vers un atlantisme novateur, ne saurait se réduire à un simple renouvellement des sources. Dans ce procès, nombreuses ont été les étapes menant à l'émergence d'une nouvelle dynamique littéraire transnationale et outre-mer.

\subsection{Une Méditerranée plus grecque que romaine la dislocation du gréco-romain}

Le concept de Méditerranée, premièrement spatial, renvoie aussi à une temporalité, celle de l'Antiquité, grecque notamment. Que la Méditerranée littéraire moderne renvoie essentiellement à la Grèce en dépit de l'incontestable socle latin des lettres européennes résulte de la complexe tradition culturelle et éducative des humanités au XIXe siècle. Ces humanités dites "classiques", qui par leur pluriel unificateur occultent la tension qui opposait le latin et le grec, sont en effet le terrain de grands antagonismes idéologiques, opposant par exemple christianisme et paganisme, romanisme et germanisme. Si le latin a toujours supplanté le grec comme matière scolaire (Chervel 1986: 3-21), il est tombé en disgrâce dans les mentalités dès le début du XIXe siècle. Ce discrédit venait d'Allemagne où, d'une part, l'on redécouvrait avec Winckelmann l'hellénisme contre le latin de l'Église et des juristes, et où d'autre part, on avait toujours récusé la médiation latine en faveur d'un lien direct entre le grec et l'allemand (Cambiano 1994: 40). Le grec a mieux résisté. C'est d'abord la langue du plus souriant paganisme. C'est aussi la langue du pays d'où proviennent les sources mentales de l'Europe. D’Allemagne également - et de Hegel plus précisément (idem: 20 ss.) - vient en effet l'idée que c'est la Grèce qui est la terre d'origine de la philosophie, et partant de tout l'imaginaire des lettres, conception d'une importance 
considérable dans la consolidation de l'image moderne de la Grèce en Europe. "Berceau", "mère", "patrie", le champ lexical de l’origine est révélateur de la fortune de cette conception dans les représentations de la Grèce au moins jusque vers la moitié du XX siècle. Lorsque par exemple Jean Moréas s'exclame, sur le mode majeur et dans le moule alexandrin “Thétis qui m’a vu naître, ô Méditerranée!” (Moreás 1907: 173), il n’identifie pas seulement la Grèce à la Méditerranée, mais associe également le lieu de sa naissance à une mer originelle de la poésie, dans la plus parfaite tradition hégélienne.

Néanmoins, dès les années 1880, la vénération obligatoire accompagnant l’héritage antique est remise en question. Les motifs de cette désaffection, sont nombreux et connus: rappelons d'abord, outre le discrédit qui frappe le latin, l'ingratitude des textes scolaires grecs qui, une fois entrés dans le canon de l'école laïque, peinent de plus en plus à transmettre le frisson qui accompagnait naguère l'étude des très païennes œuvres grecques. L'ennuyeuse sécheresse de la méthode traductive, clé de voûte de l'enseignement du grec sous la IIIe République (traditions du rendu ad verbum suivi du ad sensum) fatigue l'écolier. Les adultes ne sont pas mieux servis: comme l'atteste Marie Delcourt, les traductions du théâtre grec sont très corrompues: ${ }^{1}$

Presque toutes sont en vers, comme leurs pareilles du XVIe siècle. Le romantisme d'abord, le goût parnassien ensuite, n'ont pas été sans les atteindre. On altère le texte [...] mais pour y accentuer [...] ce qui parait "spécifiquement grec". <Ainsi>, les adaptateurs ont sollicité les textes pour y introduire le faux pittoresque, la fausse sentimentalité et la violence qui leur paraissaient indispensables à toute œuvre tragique. (Delcourt 1925: 15-16)

\subsection{La Méditerranée, mer de musée et de tourisme}

Dans ce contexte, la mer intérieure se fait lieu commun et exerce un monopole culturel tel que le résume encore Émile Zola à la fin du XIX siècle:

et il énumérait les ports de tous les pays du littoral [...] célébrant cette mer, la seule que le monde ancien eût connue, cette mer bleue autour de laquelle la civilisation a fleuri, dont les flots ont baigné les antiques villes, Athènes, Rome, Tyr, Alexandrie, Carthage, Marseille. (Zola 1891: 76) 
Les clichés que la Méditerranée véhicule débordent le texte littéraire pour apparaître aussi sur scène. Dans le traitement du décor, les chorégies de plein air connaissent un formidable engouement de 1880 à la veille de la Grande Guerre car elles peuvent être mises en scène sur des théâtres grecs et romains surplombant la Méditerranée. Dans les salles fermées, cette mer de musée et de voyage, véritable cliché visuel, est évoquée au moyen de la couleur bleue en trompe-l'œil qui pare immanquablement les toiles peintes du décor.

Ainsi expurgés, christianisés, surtraduits, platement représentés, les textes anciens engendrent une féconde lassitude, propice aux renouvellements littéraires. Comme le rappelait Jean Ballard - premier animateur des Cahiers du Sud - à propos de l'humanisme méditerranéen, "la civilisation faite par l'homme a pour fonction principale de le servir et non de l'écraser" (Ballard 1948: 7). C'est de ce poids que vont tenter de se libérer les écrivains du début du siècle. Le grief d'Apollinaire est à cet égard des plus éloquents. Il en fera l'ouverture et la clôture d'Alcools. L'incipit prend la forme de l'antithèse ParisModernité/Grèce-Rome-Antiquité:

À la fin, tu es las de ce monde ancien

Bergère ô tour Eiffel le troupeau des ponts bêle ce matin

Tu en as assez de vivre dans l'antiquité grecque et romaine (Apollinaire 1920: 7)

L'antithèse est reprise en écho dans "Vendémiaire", le dernier poème du recueil, sous la forme d'un constat: cette fois, c'est la Méditerranée elle-même, métaphorisant une Antiquité tombée en déréliction, qui reflue dans l'imaginaire du poète:

\footnotetext{
les villes du midi répondirent alors

noble Paris seule raison qui vis encore

et toi qui te retires Méditerranée

partagez-vous nos corps. (idem: 161)
}

C'est pourquoi la plupart des écrivains du début du XXe siècle tentent de se libérer de cet étouffement. Pour certains, comme les surréalistes, il s'agira d'un reflux presque total. 
La négation de l'héritage gréco-latin les conduira à s'intéresser de près à la culture celte et l'art gaulois. André Breton, fondateur et théoricien du mouvement, collectionne les monnaies celtiques, et les réhabilite comme sources esthétiques et iconographiques. Pour d'autres, la libération se fera de manière plus nuancée, en concédant un statut différent à la tradition. Les contenus canonisés des "belles lettres" sont alors revus, en grande partie sous l'impulsion de la médiation nietzschéenne, pour donner le jour à un hellénisme nouveau qui ignore à peu près la mythologie ou les Byzantins, comprend encore les grands tragiques, mais se penche surtout sur les penseurs dits présocratiques, les poètes lyriques grecs et les philosophes alexandrins.

\subsection{Cette espèce de flaque, votre Méditerranée...}

Autant que sur les contenus, la renaissance affecte le ton. L'heure n'est plus à la vénération obligée d'une tradition figée. L'espace agrandi de l'universalisme du début du $\mathrm{XX}^{\mathrm{e}}$ siècle, au sens géographique comme au sens littéraire, signifie la fin du monopole culturel exercé par la mer intérieure. Issu de ce contexte, le poète Saint-John Perse, né Alexis Leger aux Antilles, proclamera son "hostilité intellectuelle, antirationaliste [...] à l'héritage gréco-latin, et plus particulièrement latin" (Saint-John Perse 1982: XI), tout en désavouant moins les contenus des humanités classiques que leur héritage, c'est-à-dire les conditions de leur transmission. Le ton ne peut être que celui d'une irrévérence nietzschéenne, qui préside au renversement des valeurs traditionnelles. Tous les attributs de la Grèce que naguère on admirait sont déconstruits, avant que d'être réinventés.

La Méditerranée, symbole majeur, ne fait pas exception. Ses dimensions, son apparence, sa couleur, son odeur, son mouvement, ses contenus, sa température, tout y passe.

Julien Gracq, dans Liberté Grande (1990: 44), dénonce l'immobilisme stérile de "La Méditerranée plate dont le blanc des vagues semble toujours fatigué d'un excès de sel", produit d'un surcroît malsain. Avec sa critique implicite de la Méditerranée homérique auxmille-vagues, l'invalidation de Gracq vise la tradition antique. Ces contenus fatigués, vidés de leur pouvoir d'évocation, ces attributs éculés composent désormais un grand dépotoir: 
c'est en ces termes que Paul Claudel inventorie "toutes les marques de la Méditerranée, marmites craquées, vieux couteaux, fanaux, accordéons, astrolabes, épissoires, figures de proue" (Claudel 1927: 363). Ce que reproche surtout Claudel à la Méditerranée, lui qui a sillonné le Pacifique et a accordé à cet océan une place éminente dans sa poétique, c'est d'être petite, une "espèce de flaque" (Claudel 2011: 893), inapte à contenir les limites de la nouvelle littérature. Étriquée, la mer ancienne semble dorénavant dépourvue du mobilisme héraclitéen qui anime une grande partie de la littérature moderne. Désormais, la Méditerranée se verra opposer "la vaste chaussée océane" (Saint-John Perse 1982: XLI) pour ses dimensions spatiales et mentales dilatées. Sa couleur bleue enfin, marque identitaire dépassée qui engendre l'écœurement, produira un contre-symbole esthétique.

\section{Du celtisme à l'atlantisme littéraire}

Ainsi que le résume Michel Pastoureau, la perception de la couleur et son utilisation sont toujours culturelles et idéologiques, relevant "d'enjeux économiques, politiques, sociaux, symboliques s'inscrivant dans un contexte précis", de sorte que "toute histoire des couleurs doit d'abord être une histoire sociale" (Pastoureau 2008: 17). Quelle sémiotique des couleurs se lit-elle dans le passage du bleu méditerranéen au vert océanique?

\subsection{Bleu méditerranéen contre vert océanique}

Le bleu méditerranéen, renforcé par un recours abusif à ce poncif de la littérature de voyages, est omniprésent dans la littérature française de la première moitié du XXe siècle. Le recensement des occurrences ${ }^{2}$ de "mer bleue", avec toutes les variations possibles, devient très vite fastidieux. Outre la "mer bleue autour de laquelle la civilisation a fleuri" de L'Argent d'Émile Zola, on recense trois "mer bleue" dans les seuls Contes et Nouvelles de Maupassant; plusieurs autres dans les récits de Loti, qui la qualifie par ailleurs d"'éternellement bleue", d'"invraisemblablement bleue", et qui voit en la Méditerranée une "chose bleue qui ne finit plus"; suivent André Gide, Anatole France, Louis Aragon... Ce grand bleu conventionnel que les écrivains du XXe siècle se feront un devoir de dépouiller de ses trop directes connotations, va progressivement virer sous le regard corrosif des nouvelles 
générations. Dans le premier récit de voyage Albert t’Serstevens, L'Itinéraire Espagnol (1933), la condamnation est devenue franche: le bleu est "sentimental" et "conventionnel". Par ce double reproche, t’Serstevens dénonce à la fois le poids écrasant de la tradition et l'attitude obligée de respect du symbole.

Chez Saint-John Perse, poète par excellence de l'exaltation marine du second $\mathrm{XX}^{\mathrm{e}}$ siècle (Amers date de 1957), ${ }^{3}$ verte est devenue la mer, bien qu'elle procède "d'une même vague depuis Troie" (Saint-John Perse 1982: p. 326, 339-340). Tout en gardant la référence grecque, Saint-John Perse renouvelle la tradition de la catena aurea en la déclinant sur le mode maritime. Peu d'occurrences de bleu dans cette œuvre pourtant nourrie de Grèce, contre plus d'une centaine de verts: "la Mer, immense et verte" (idem: 259), "La mer louable ouvrait ses blocs de jaspe vert" (idem: 274), "La mer aux spasmes de méduses menait, menait ses répons d'or, par grandes phrases lumineuses et grandes affres de feu vert" (ibidem). Enfin, on appréciera dans cette dernière citation de l'Amant d'Amers la contiguïté délibérée de motifs grecs et d'une mer verte:

Et la mer entre nous ne trace point frontière... Nous irons sur la mer aux très fortes senteurs, l'obole de cuivre entre les dents. L'amour est sur la mer, où sont les vignes les plus vertes; et les dieux courent aux raisins verts, les taureaux aux yeux verts chargés des plus belles filles de la terre. (idem: 355)

Les références antiques sont réactualisées dans la texture du poème après avoir été banalisées par les pluriels amplificateurs et l'absence de noms propres. Bien que l'on puisse reconnaître dans ce passage le rituel préludant au passage du fleuve des Enfers, l'enlèvement d'Europe par Zeus métamorphosé en taureau, la présence de Dionysos et,

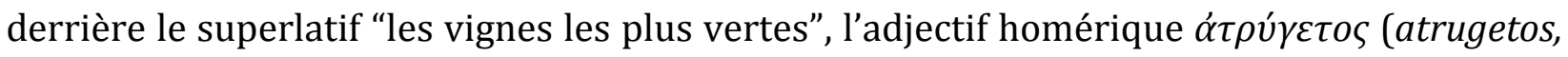
qui ne peut être vendangée) qualifiant traditionnellement la mer (Hurst 1979: passim), la référence grecque n'a plus le statut de clé indispensable à la compréhension du passage: tel était le parti du poète par-devant l'héritage hellénique. De même, le remplacement délibéré et réitéré du bleu par la couleur verte ne saurait être imputé à une volonté de restituer une quelconque vérité "philologique", de remplacer le bleu inexistant chez les Grecs et les Latins 
par la couleur qui lui en serait le plus proche. La contre-référence de la couleur verte, sémantiquement et symboliquement chargée, est cependant loin de constituer une génération sémiotique spontanée. Elle est trouvée, ou plutôt retrouvée, dans la tradition du celtisme littéraire du XIX ${ }^{\text {e }}$ siècle, entre autres redécouverte par les surréalistes, comme on l'a vu plus haut. Comme naguère Madame de Staël, Chateaubriand, Balzac ou Hugo, on oppose au XXe siècle le Nord et le Sud, l'Atlantique vivifiant à l'insalubre Méditerranée. Montherlant le fera dire sans ambages à l'un de ses personnages: "Au Havre [...], le vent de la mer ne m'a jamais rendue malade comme la chaleur noire de cette Méditerranée" (Montherlant 1943: 295).

\section{Le continent atlantique comme creuset identitaire}

Saint-John Perse, qualifié de "surréaliste à distance" par Breton, ira plus loin dans l'élaboration d'une poétique maritime originale. Il précise, dans sa "Biographie" rédigée à la troisième personne qui inaugure le volume de ses CEuvres Complètes dans la Pléiade, que son hostilité intellectuelle à l'héritage latin, "tient à ses affinités celtiques, qui sont profondes en lui" (Saint-John Perse 1982: XL). Ainsi la prédominance du vert sur le bleu traduira-t-elle dans sa poétique la victoire du celtisme sur le méditerranéisme, où il s'agit bien moins d'un celtisme linguistique que géographique: les Antilles natales du poète au climat océanique lui permettent d'opposer, au plan poétique, l’ombre créatrice et la lumière aveuglante. En 1956, Saint-John Perse avoue dans une lettre à Alain Bosquet son "horreur de la Méditerranée", ajoutant: "tout ce qui est celte en moi s'y oppose violemment" (Bosquet 1990: 295).

En 1957, lorsqu'il revient pour la première fois en France après l'exil américain de la guerre et s'installe en Provence, il rappelle cette horror mediterranei dans une autre lettre:

Un peu d'insolence secrète saura calmer en moi ces inquiétudes irraisonnées [...]. La lumière méditerranéenne fera le reste, en m'aveuglant à souhait. Ne jamais trop interroger nos nuits, même en pays si clair. (Terre d'archaïsme et de mythologie [...] sous le signe trop connu de la petite chouette d'Athênê.)

À la Méditerranée comme mer, m’habituerais-je jamais? (Saint-John Perse 1982: 924) 
Un an plus tard, toujours dans sa correspondance:

Je dois faire face à cette mer latine qui n'est point celle de mon enfance [...]. Je n'en perçois que mieux le Celte en moi, à cette rumeur lointaine qui me descend toujours du Nord par l'oreille interne. (idem: 1059)

L'aversion est donc à la fois celle de l'enfant des mers vertes antillaises et celle d'un poète rebuté par les symboles éculés de la civilisation méditerranéenne. Saint-John Perse inscrit enfin son hypostase atlantique à la fin de la section récrite des "Lettres d'Asie" de sa correspondance, en la reliant expressément à l'espace celte. Sa lettre adressée au magistrat tibétologue Gustave-Charles Toussaint se termine sur ces mots "Si vous rentrez avant moi en Europe, saluez pour moi votre Bretagne natale. Elle a toujours été terre sainte pour ceux qui, comme moi, comptent trois siècles d"hommes d'Atlantique'” (idem: 896). Par une déclaration solennelle, Saint-John Perse atteste ici sa volonté de "clore ce chapitre de sa vie par cette appellation hautement revendiquée d'hommes d'Atlantique'” (Mayaux 1994: 172).

Mais pour le grand commis de la République française qu'était Alexis Leger, seul l'espace poétique pouvait pendre acte d'une mutation de la nationalité française en une identité atlantique, en une nature essentielle transnationale. Et encore, cela ne se fera que sur le mode occulte.

\section{1. "Né d'Atlantique"}

La dédicace inscrite par Saint-John Perse au fronton de son recueil poétique Vents, publié aux États-Unis en 1946, “Pour Atlanta et Allan P.” (Saint-John Perse 1982: 175) fut longtemps mystérieuse. Il a été montré ailleurs (Chehab 2007: passim) qu'elle constitue en réalité un cryptonyme de plus de l'identité plurielle du poète qui lui permet, sous une forme apparemment canonique, de se dédier Vents à soi-même dans la tradition, renouvelée par Walt Whitman, de l'auto-dédicace de l'école des Stoïciens. ${ }^{4}$

Si le dédicataire "Allan" fait clairement office de double du Poète pour avoir été l’un 
de ses surnoms d'enfance, la composante féminine du faux couple de dédicataires, Atlanta, sans autre référent réel, renvoie avec insistance à "Atlantique", espace mental privilégié du jeune Alexis Leger qui sera ultérieurement érigé en concept identitaire par Saint-John Perse. De cela témoigne à escient la "Biographie" déjà mentionnée du poète, rédigée par ses soins à la troisième personne. L'expression "être un homme atlantique" y donne lieu à un long développement, dont voici la fin:

À la question: “D’où êtes-vous, de quel pays?" ils n'eussent point répondu: “De telle ou telle île”, mais: "D’Atlantique". Un Saint-Leger Leger naissait d'Atlantique comme on naît d'Europe ou d'Amérique. Il y reconnaîtrait toujours le masque de son destin. (Saint-John Perse 1982: XL-XLI)

La mystérieuse Atlanta se décode ainsi comme une personnification du principe atlantique, féminisé pour s'adapter au paradigme dédicataire. Sa fonction: entériner au plus près de l'œuvre l'"atlanticité" fondatrice de l'identité du poète.

\subsection{L'insularité du continent atlantique}

Il a été dit plus haut que le celtisme identitaire revendiqué par Saint-John Perse n'était pas linguistique mais géographique. Plus encore que ses brumes climatiques se transposant en accès lyriques, le celtisme européen propose ses territoires morcelés en îles, presqu'îles et péninsules, qui se prêtent à l'identification analogique avec les Antilles, ou "terres-d'avant-le-continent", selon la formule de Patrick Chamoiseau (1993: 37). De fait, Saint-John Perse va partager avec Édouard Glissant une poétique archipélique, et exhausser l'insularité biographique au rang d'insularité mythographique.

\subsection{Lieux de naissance: îles}

Né sur l'îlet de "Saint-Leger-les-Feuilles"

Saint-John Perse

Sans doute un îlet en rade d'un port est-il le plus sûr gîte de l'errance.

L'îlet-les-Feuilles, dans le port de Pointe-à-Pitre Édouard Glissant 
On ne peut s'y tromper: l'île dans l'imaginaire poétique de Saint-John Perse et d’Édouard Glissant est bien plus mythique que biographique. Lorsque Saint-John Perse indique le lieu de sa naissance dans sa "Biographie" par ces mots brefs "Né sur l'îlet de 'Saint-Leger-les-Feuilles'”' (Saint-John Perse 1982: p. IX), ce n'est pas par souci d'exactitude référentielle, mais pour revendiquer une extra-territorialité poétique face au Vieux continent. Cette brève information résume tout un programme, savamment distribué dans le volume des CEuvres Complètes, et qu'il appartient au lecteur de reconstituer à partir de son dispersement archipélique. On trouve par exemple dans l'apparat de notes du volume de la Pléiade un curieux développement, où une péjoration déguisée est adroitement compensée et se transmue en déclaration d'appartenance:

L'insignifiante petite île, ou "îlet" privé, où je suis né, n'était qu'une propriété de plaisance appartenant à ma famille paternelle depuis le XVIII ${ }^{e}$ siècle; elle est loin de mériter la considération qu'on voudrait lui prêter dans ma légende littéraire.

Modeste îlot de quelque 3 hectares, l'îlet "Saint-Leger-les-Feuilles" n'a même jamais eu de statut géographique propre, étant du ressort administratif du port de Pointe-à-Pitre [...]. Si bien, que, né en fait dans cette petite île d'agrément, je me trouve, "administrativement", né dans la ville de Pointe-àPitre, où ma naissance fut officiellement déclarée.

L'îlet en question [...] diffère grandement des îles voisines [...]. Ce caractère exceptionnel d'île rocheuse et fortement boisée [...] lui avait valu sur les vieilles cartes [...] le nom d'"îlet-à-Feuilles" ou d'”̂le Feuilles". C'est ma famille paternelle (Saint-Leger Leger) qui lui adjoignit, en l'acquérant, le nom patronymique de Saint-Leger, d'où l'appellation de "Saint-Leger-les-Feuilles". (Saint-John Perse 1982: 1087)

L'importance de cet îlot dans la poétique persienne n'échappe pas à Édouard Glissant, qui écrit dans son commentaire sur Saint-John Perse:

Sans doute un ilet en rade d'un port est-il le plus sûr gîte de l'errance. L'Ilet-les-Feuilles, dans le port de Pointe-à-Pitre. Un ilet dans l'ancrage d'une île, délimité non par ses plages de sable rouies de mangrove mais par l'écriture des hauts navires. (Glissant 1997a: 431)

En effet, l'îlet qui, comme le rappelle Chamoiseau, s'appelle lilèt en langue créole et désigne de "minuscules concrétions quasi inhabitables" (Chamoiseau 1993: 38), constitue 
un degré zéro d'espace habitable, un lieu irréductible assimilable, selon la topoanalyse bachelardienne, à un espace du "moi", au royaume insulaire qu'est aussi tout espace identitaire. Il faut se souvenir ici que le premier nom de plume d'Alexis Leger est "Saintleger Leger", où résonne encore le nom de lîlet "Saint-Leger-les-Feuilles". On le retrouve aussi dans les premiers poèmes: à l'âge de vingt ans, le poète publie le recueil Pour Fêter une Enfance avec une épigraphe cryptique en anglais: King Light's Settlements (Les Établissements du Roi Leger), qui renvoie encore à son îlet. Par ces subtils va-et-vient entre la patronymie et la toponymie, le nom devient lui-même une île identitaire où le poète élit domicile, comme on peut le voir dans le poème "Exil": "J'habiterai mon nom", fut sa réponse aux questionnaires du port (Saint-John Perse 1982: 135).

Clausule à laquelle fera écho Édouard Glissant dans le Traité du Tout-monde:

Ces noms que j'habite s'organisent en archipels. Ils hésitent aux bords de je ne sais quelle densité, qui est peut-être une cassure, ils rusent avec n'importe quelle interpellation, qu'ils débordent infiniment, ils dérivent et se rencontrent sans que j'y pense. (Glissant 1997b: 77)

Les archipels identitaires et poétiques de Saint-John Perse le premier, puis d'Édouard Glissant, se définissent en rupture avec les symbolismes traditionnels qu'ils dénoncent. Le mot même, avant la chose, puisque "pas une seule fois dans sa Biographie ou dans ses CEuvres Complètes Saint-John Perse n'emploie le mot 'archipel' [...] il ne connaît que 'les îles' et l'entr'îles'” (Claverie 2003: 296). Cela s'explique du point de vue purement référentiel d'abord, car lorsque l'on mentionnait, en français, les 'îles', sans autre prédicat, il s'agissait des Antilles. D'un autre côté, lorsque l'on mentionnait l'Archipel' sans autre forme de prédication non plus, cela renvoyait à la culture antique, et symbolisait depuis Hölderlin l'apport culturel hellénique. Pour les défenseurs d'une nouvelle lecture des textes classiques que furent Saint-John Perse et Édouard Glissant, l'archipélité hellénique se devait d'être détrônée de son piédestal humaniste et romantique, avant que d'être resémantisée. Comme l'écrit Saint-John Perse:

Derrière l'écran lumineux du site méditerranéen, c'est toute une ambiance atmosphérique et tout un 
arrière-plan psychologique, toute une imprégnation d'histoire et de civilisation ancienne à laquelle le poète demeure étranger. (Saint-John Perse 1982: XL)

Outre l'archipel grec saturé de connotations, les deux poètes dénoncent, quoique différemment, l'imaginaire collectif occidental de l'archipel, encore largement tributaire des utopies colonisatrices des siècles passés. Alors que les Antilles étaient perçues par l'Européen comme des "terres-d'avant-le-continent, sortes de paliers, marchepieds" (Chamoiseau 1993: 38), ses habitants ne voyaient pas les îles comme des entités indépendantes, mais comme "des émergences d'un habitat archipélique" (ibidem), d'un espace couvrant l'ensemble de l'archipel et où la mer lie plus qu'elle ne sépare. Saint-John Perse le souligne encore dans sa "Biographie" qui fait décidément office d'acte déclaratif d'allégeance identitaire:

Si importante et décisive fut l'influence du fait atlantique dans la formation humaine des premiers Antillais français, que leurs fils des Îles, tenant géographiquement l'Atlantique pour un "continent" plus que pour une "mer", y virent plus un "habitat" qu'un environnement. (Saint-John Perse 1982: XL)

Ainsi l'ensemble archipélique s'oppose-t-il en continent à cet agrégat d'îles isolées des utopies insulaires colonialistes. Car l'île vierge et heureuse du discours touristique provient autant du mythe des Bons sauvages des XVII e et XVIIIe siècles, que de l'Utopie colonisatrice "basée sur l'idéal de l'île passive, du rocher transparent et de l'espace maîtrisable" (Dash 1999: 18), celle d'un Robinson Crusoé. La platitude de ce discours masque l'épaisseur, la profondeur qui se cache sous sa surface. Le Blanc des îles, le béké Saint-John Perse, parle en initié de la "vaste chaussée océane" (Saint-John Perse 1982: XLI), et rencontre par là les poètes anglophones des Îles lorsqu'ils déclarent que l'unité est sousmarine (Chamoiseau 1993: 38). Chez Glissant, c'est l'histoire qui se transmue en poétique, où le système relationnel souterrain rompt la vision occidentale d'une archipélité de surface. Pour le Martiniquais, l'épiderme de la mer cache un tapis de cadavres: ceux des Noirs jetés par-dessus bord par les négriers lorsqu'ils étaient acculés par les bateaux les poursuivant après l'interdiction de la Traite. 
L'île colonialiste et touristique, utopiste et utilitaire, limitée à sa surface, est un isolat. Comme résume Chamoiseau, là où les Caraïbes "naviguaient sans cesse d'île en île, de rivage en rivage, au gré de leurs fêtes, de leurs alliances", là où la mer liait et reliait, précipitait en contact, le "colon européen, lui, s'isole lui-même dans l'île: il est en rivalité avec d'autres puissances colonialistes, il dresse des remparts, dessine des frontières, des couleurs nationales [...]. Il porte dans sa tête les barreaux de l'exil" (ibidem). Or, l'idée de frontière telle que nous la concevons aujourd'hui naît au XVIIIe siècle elle aussi, "au moment où se forge le sentiment de l'identité nationale" (Martinière/ Le Ménahèze 2003: 7). Dans la littérature, avant les sonores déclarations de Hugo dans la préface de Cromwell appelant à l'abolition des frontières séparant les genres littéraires, des voix se font entendre au XVII siècle pour réclamer des possibilités de passage d'une forme à l'autre. La poétique archipélique, elle, ne connaît pas ces frontières absolues que d'un pas on traverse. Ses limites sont enchevêtrées, complexes, ses plages étendues. Aussi bien entre lîle et la mer qu'entre la surface et la profondeur, grand est l'interrègne et inépuisable le territoire des ouvertures et des possibles. Cette poétique de l'entr'îles donne lieu, depuis le banyan de Claudel à la mangle de Glissant, à toute une rhétorique du rhizome, de la plage, de lîle mangrove. Espaces mixtes et intermédiaires, riches et ouverts, ils abolissent toute idée de solitude du circonscrit.

Les œuvres de Saint-John Perse et d'Édouard Glissant se font signe, chaque île-texte communique avec ses sœurs tout en gardant intacte son irréductibilité: la mer mouvante en est le lien, et le terrain du désir qui renvoie à l'espace matriciel caribéen. Pour Saint-John Perse, "La mer, entre les îles, est rose de luxure" (1982: 49), elle est lieu créateur de transgressions, de coexistence des contraires et de transcendance de l'esprit:

Mer utérine de nos songes et Mer hantée du songe vrai,

$[\ldots]$

Ô toi qui sais et ne sais pas, ô toi qui dis et ne dis pas,

$[\ldots]$

Ô Consanguine et très lointaine, ô toi l'inceste et toi l'aînesse,

$[\ldots]$ 
Mer à jamais irrépudiable, et Mer enfin inséparable!

$[\ldots]$

Est-ce toi, Nomade, qui nous passeras ce soir aux rives du Réel? (idem: 380)

Le nomadisme poétique à l'œuvre dans ce passage exalte la non-sédentarisation du poète et de la poésie. La grande question qui se pose pour la poétique de l'insularité est celle de relation ou de solitude, de dispersion ou de cohésion, d'ouverture ou de fermeture, tant "l'idée d'archipel conjoint deux notions contradictoires: l'isolement de l'île et la liaison de l'ensemble" (Joubert 2003: 318). Or l'histoire du mot se retrouve dans l'opposition de ses acceptions actuelles: un archipel c'était d'abord une mer, signifiant, selon l'étymologie $\alpha \rho \chi \iota \pi \varepsilon ́ \lambda \alpha \gamma o \varsigma$ mer ancienne ou mer par excellence, voire une mer Égée déterritorialisée, mais une mer - quoique parsemée d'îles - où la continuité est mise au premier plan. Aujourd'hui, un archipel est un groupe d'îles, ce qui met l'accent sur son caractère discontinu (idem: 319). Si pourtant lîle persienne est souvent synonyme de solitude, c'est davantage d'un solipsisme philosophique qu'il s'agit que de fermeture littéraire, puisque sa poésie s'épuise dans l'espoir universaliste d'un nouvel humanisme. Dans une lettre SaintJohn Perse déclare: "Peut-être la notion d'espace se confond-elle un jour avec celle d'insularité, où, pour un Antillais, s'abîme toute solitude" (Saint-John Perse 1982: 836-837). Et dans son grand poème Vents, l'archipel est formé d'une pluralité de solitudes qui aboutit à l'aporie du néant:

... Plus loin, plus loin, où sont les premières îles solitaires - les îles rondes et basses, baguées d'un infini d'espace, comme des astres -

... Plus loin, où sont les îles hautes

... Et au-delà, les purs récifs, et de plus haute solitude

... Et au-delà et au-delà, sont les derniers froncements d'humeur sur l'étendue des mers. Et mon poème encore vienne à grandir avec son ombre sur la mer ...

... Et au-delà, et au-delà, qu'est-il rien d'autre que toi-même -qu'est-il rien d'autre que d'humain ? Minuit en mer après Midi ... Et l'homme seul comme un gnomon sur la table des eaux... Et les capsules de la mort éclatent dans sa bouche. (idem: 238) 
Espaces épiphaniques, l'île, l'entr'îles et leur vaste chaussée souterraine sont le lieu où la langue poétique s'ouvre à "la promesse d'îles" persienne et à "la pensée archipélique" glissantienne.

\section{NOTES}

1 Il faudra attendre les traductions de Jean Bollack, par exemple, pour leur donner un souffle vraiment contemporain.

${ }^{2}$ Effectué sur la base Frantext.

${ }^{3}$ Avec son très polysémique titre Amers, qui entendait offrir par la poésie de nouveaux repères humanistes au lendemain de la Seconde Guerre, Saint-John Perse renouvelle un ancien jeu sémantique de la littérature européenne:

Tristan dit à voix basse: 'Ah! belle, dites-le-moi, qu'est-ce donc, qui vous tourmente?' Yseut répondit: 'L'amer est mon tourment: c'est l'amer qui m'oppresse l'âme, l'amer qui me fait mal'. Comme elle répétait si souvent l'amer, Tristan chercha avec soin ce qu'elle entendait par ce mot. Il put bien comprendre que l'amer signifiait l'aimer, l'amer, la mer. (von Straßburg †1210: vers 11.986-12.019)

Ce jeu ancien, que Gottfried von Straßburg cite en français, remonte au manuscrit de Thomas d'Angleterre, fait récemment confirmé par la découverte du fragment de Carlisle. Voir Michael Benskin, Tony Hunt, Ian Short (1992), "Un nouveau fragment du Tristan de Thomas", Romania, tome 113 n451-452, 1992, p. 289-319. Voir également le sonnet baroque "À Philis" de Pierre de Marbeuf (1596-1645), qui reproduit le même jeu: "Et la mer et l'amour ont l'amer pour partage, / Et la mer est amère, et l'amour est amer [...]".

4 Pour Hadot, traducteur des Pensées de Marc Aurèle, il ne fait pas de doute que la traduction française plus

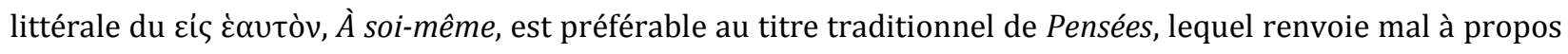
aux Pensées de Pascal. Dans sa liste des différents titres que l'œuvre a reçus, on retient, avec lui, les traductions qui ont choisi la version littérale: L'editio princeps d'abord, qui proposait le titre grec Tò zís غ̇autòv. Puis, en latin, "de seipso ad seipsum", en anglais "To himself", en français "À moi-même" ou "À soimême". Hadot ajoute que le titre de Marc-Aurèle n'innove pas, qu'il décrit un genre littéraire déjà attesté dans l'histoire de la littérature exhortative grecque, laquelle commence en effet avec Solon, le premier utilisateur du titre ̀̀ moi-même. 


\section{Bibliographie}

Apollinaire, Guillaume (1920), Alcools, Paris, NRF.

Ballard, Jean (1948), Permanence de la Grèce, Paris, Le Cahiers du Sud.

Benskin, Michael / Tony Hunt / Ian Short (1992), "Un nouveau fragment du Tristan de Thomas", Romania, t. 113, n451-452, 1992, p. 289-319.

Bosquet, Alain (1990), La Mémoire ou l'Oubli, Paris, Grasset.

Cambiano, Guiseppe (1994), Le Retour des Anciens, Paris, Belin.

Chamoiseau, Patrick (1993), "Chanter l'Île”, Nouvel Observateur, HS 18, 36-38 (colonnes a, b et c).

Chehab, May (2007), “Atlanta et Allan P.”, La Nouvelle Anabase, no 3, Paris, L'Harmattan, 1222.

-- (2003), "La Méditerranée et la Désaffection de l'Antique", Méditerranée: Ruptures et Continuité, no 37, Lyon, Travaux de la Maison de l'Orient Méditerranéen, 35-41.

Chervel, André (1986), Les Auteurs Français, Latins et Grecs au Programme de l'Enseignement Secondaire de 1800 à nos Jours, Paris, IRNP.

Claudel, Paul (2011), Le Soulier de Satin, Théâtre, vol. II, Paris, Gallimard.

-- (1927), Protée, Paris, Gallimard.

Claverie, André (2003), "L'Archipel Oublié de Saint-John Perse", in L'Imaginaire de l'Archipel, Paris, Karthala, 295-303.

Dash, Michel (1999), "Île Rocher/Île Mangrove", in Poétiques d'Édouard Glissant, Paris, Presses de l'Université de Paris-Sorbonne, 17-24.

Delcourt, Marie (1925), Étude sur les Traductions des Tragiques Grecs et Latins en France 
depuis la Renaissance, Bruxelles, Marcel Hayez.

Glissant, Édouard (1997a), Le Discours Antillais, Paris, Gallimard, [1981].

-- (1997b), Traité du Tout-Monde, Paris, Gallimard.

-- (1996), Introduction à une Poétique du Divers, Paris, Gallimard.

Gracq, Julien (1990), “Les Nuits Blanches”, in Liberté Grande, Paris, Corti [1946], p. 284-285.

Hurst, André (1979), "Sur un Passage d'Amers: La Culture Grecque comme Élément de Savoir Intégré", in Espaces de Saint-John Perse 1-2, Aix-en-Provence, Publications de l'Université de Provence, p. 213-227.

Joubert, Jean-Louis (2003), "L'Archipel Glissant”, in Voisset, Georges (dir.), L'Imaginaire de l'Archipel, Paris, Karthala, 317-323.

Martinière, Nathalie/ Sophie Le Ménahèze (2003), Écrire la Frontière, Limoges, Presses Universitaires de Limoges.

Mayaux, Catherine (1994), Saint-John Perse: Les Lettres d'Asie, Cahiers Saint-John Perse, vol. 12, Paris, Gallimard.

Montherlant, Henri de (1943), Le Fils de Personne, Paris, Robert Laffont.

Moréas, Jean (1907), Poèmes et Sylves, Paris, Mercure de France.

Pastoureau, Michel (2008), Noir: Histoire d'une Couleur, Paris, Éditions du Seuil. Saint-John Perse (1982), CEuvres Complètes, Paris, Gallimard.

von Straßburg, Gottfried (†1210), Tristan und Isolde, vers 11.986-12.019, en français dans toutes les éditions.

Zola, Émile (1891), L'Argent, Paris, Charpentier et Fasquelle. 
M. Chehab est Professeur de littérature française et comparée à l'Université de Chypre. Chercheur invitée de l'Université de Cergy-Pontoise (2014), Visiting Research Fellow de l'Université de Princeton (2005), Professeur Jean Monnet d'histoire de l'intégration européenne (2003-2006), elle a publié Saint-John Perse, neveu de Nietzsche (Champion, 2009), “De l'Histoire Longue de la Culture à l'Histoire Courte de l'Imposture: La Lecture biaisée de Fernand Braudel par Samuel P. Huntington" in Mayaux, C. (ed.) (2011), Écrivains et Intellectuels Français Face au Monde Arabe, 261-271, et co-dirigé Méditerranée: Ruptures et continuité, 2003. 